\title{
Extramedullary blast crisis as initial presentation in chronic myeloid leukemia with the e1a2 BCR-ABL1 transcript: A case report
}

\author{
DI AI ${ }^{1,2}$, WEI LIU ${ }^{1,3}$, GARY LU $^{1}$, KEYUR PRAVINCHANDRA PATEL ${ }^{1}$ and $\mathrm{ZI} \mathrm{CHEN}^{1,4}$ \\ ${ }^{1}$ Department of Hematopathology, The University of Texas MD Anderson Cancer Center, Houston, TX 77030; \\ ${ }^{2}$ Department of Pathology, Scott and White Memorial Hospital, Texas A\&M Health Science Center, Temple, TX 76508, USA; \\ ${ }^{3}$ Department of Pathology, The First Affiliated Hospital of Soochow University, Suzhou, Jiangsu 215006; \\ ${ }^{4}$ Department of Hematology, Huashan Hospital, Fudan University, Shanghai 200040, P.R. China
}

Received July 8,2015; Accepted August 5, 2015

DOI: $10.3892 / \mathrm{mco} .2015 .641$

\begin{abstract}
A 23-year-old woman presented with enlarged right inguinal lymph nodes. The pathological examination of the nodes revealed infiltration by myeloid sarcoma. A bone marrow smear and biopsy revealed cytogenetic abnormalities, with $46, \mathrm{XX}, \mathrm{t}(9 ; 22)$ and chronic myeloid leukemia (CML) was diagnosed. The ela2 BCR-ABL1 fusion transcript was detected. The patient received imatinib-based combined chemotherapy, allogeneic hematopoietic stem cell transplantation, donor lymphocyte infusions and dasatinib treatment. The patient achieved complete response and has remained leukemia-free for $>48$ months. To the best of our knowledge, this is the first case report of CML with the ela2 BCR-ABL1 transcript, with extramedullary blast crisis as the initial presentation. The aim of the present study was to discuss this special case with reference to the literature.
\end{abstract}

\section{Case report}

A 23-year-old woman presented with enlarged right inguinal lymph nodes during her seventh month of pregnancy in December, 2005. The patient had no fever, chills, bleeding, dizziness, facial pressure or hepatosplenomegaly. A review of the remainder of the organ systems yielded unremarkable findings. The past medical history was also unremarkable. A fine-needle aspiration of the inguinal nodes was performed and abundant lymphoid cells with a few scattered histiocytoid cells were found, suggesting a lymphoproliferative disorder.

Correspondence to: Dr Zi Chen, Department of Hematology, Huashan Hospital, Fudan University, 12 Urumqi Middle Road, Shanghai 200040, P.R. China

E-mail: zchen7@mdanderson.org

Key words: bone marrow transplant, chronic myeloid leukemia, ela2 transcript, extramedullary blast crisis, targeted therapy
The patient then underwent an excisional biopsy of an enlarged right inguinal node. The histological examination revealed effacement of the architecture, with prominent expansion of interfollicular areas by a neoplastic infiltrate composed of blasts cells with a high nucleus:cytoplasm ratio, slightly irregular nuclei, fine chromatin and small nucleoli. Numerous mitotic figures were present. The immunohistopathological examination demonstrated that the neoplastic cells were myeloblasts with aberrant T-cell antigen expression: $\mathrm{MPO}^{+}$, $\mathrm{CD}^{+}, \mathrm{CD}^{+}, \mathrm{CD}^{+}{ }^{+}, \mathrm{CD} 4^{+}, \mathrm{CD}_{4}{ }^{+}, \mathrm{TdT}^{+}, \mathrm{Bcl}-2^{+}, \mathrm{CD}^{2} \mathrm{a}^{-}$, CD2-, CD3-, CD4-, CD8-, CD20-, CD45RO', CD56-, CD57-, Bcl-1', Bcl-6 ${ }^{-}$and Pax-5- The pathological examination of the lymph nodes revealed infiltration by myeloid sarcoma (MS). The peripheral blood cell count and differentiation were within the normal range. A subsequent bone marrow (BM) aspiration was performed, revealing a hypercellular BM $(\sim 70 \%$ cellularity), without histomorphological evidence of lymphoma or leukemia, although trilineage hematopoiesis with myeloid hyperplasia was observed. The BM smear results were as follows: blasts $2 \%$, progranulocytes $0 \%$, myelocytes $21 \%$, metamyelocytes $19 \%$, granulocytes $20 \%$, eosinophils $2 \%$, basophils $0 \%$, lymphocytes $11 \%$, plasma cells $1 \%$, monocytes $2 \%$ and normoblasts $22 \%$. BM flow cytometry detected no significant increase in blasts or abnormal cells. The T-, Band NK-cell populations were normal in number. Interestingly, however, the karyotype analysis of BM detected 46,XX,t(9;22) (q34;q11.2)[15]/46,XX[5] (Fig. 1) and the fluorescence in situ hybridization (FISH) test result was positive for $B C R-A B L 1$ rearrangement (116/200) (Fig. 2). The peripheral blood counts remained normal.

In March 2006, after the delivery of the fetus, BM aspiration was repeated. The BM biopsy revealed $70 \%$ cellularity, whereas the morphology of megakaryocytes was unremarkable. The BM smear revealed $2 \%$ blasts and slightly left-shifted granulocytes. The karyotype analysis of BM detected 46,XX,t(9;22)(q34;q11.2)[13]/46,XX[7] and FISH confirmed $B C R-A B L 1$ rearrangement (129/200). Quantitative polymerase chain reaction (PCR) analysis of the peripheral blood identified the ela2 BCR-ABL1 fusion transcript. The percentage of $B C R-A B L 1$ to $A B L 1$ transcripts was 66.78 . 


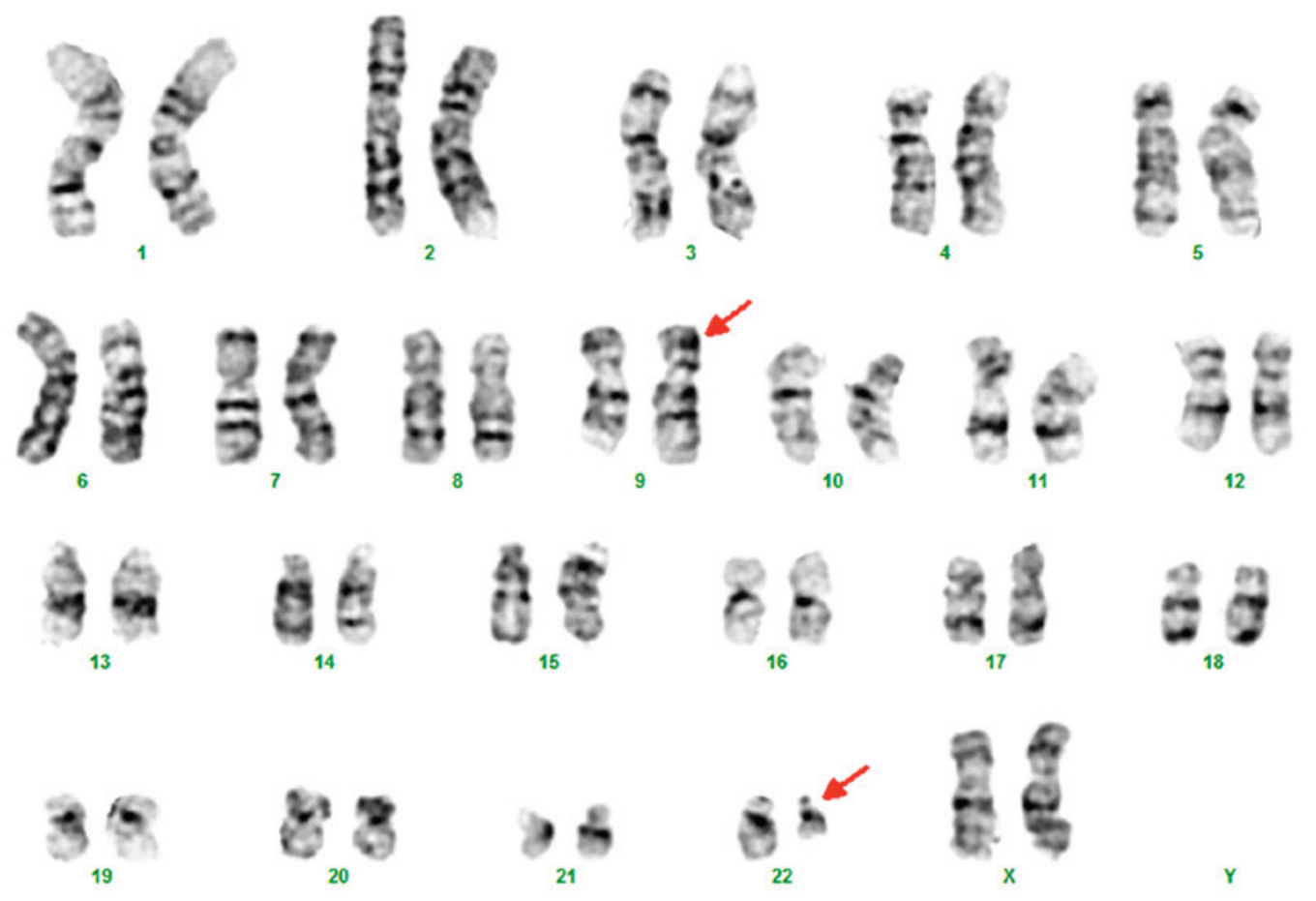

Figure 1. Cytogenetic analysis by G-banding identified 46,XX,t(9;22)(q34;q11.2).

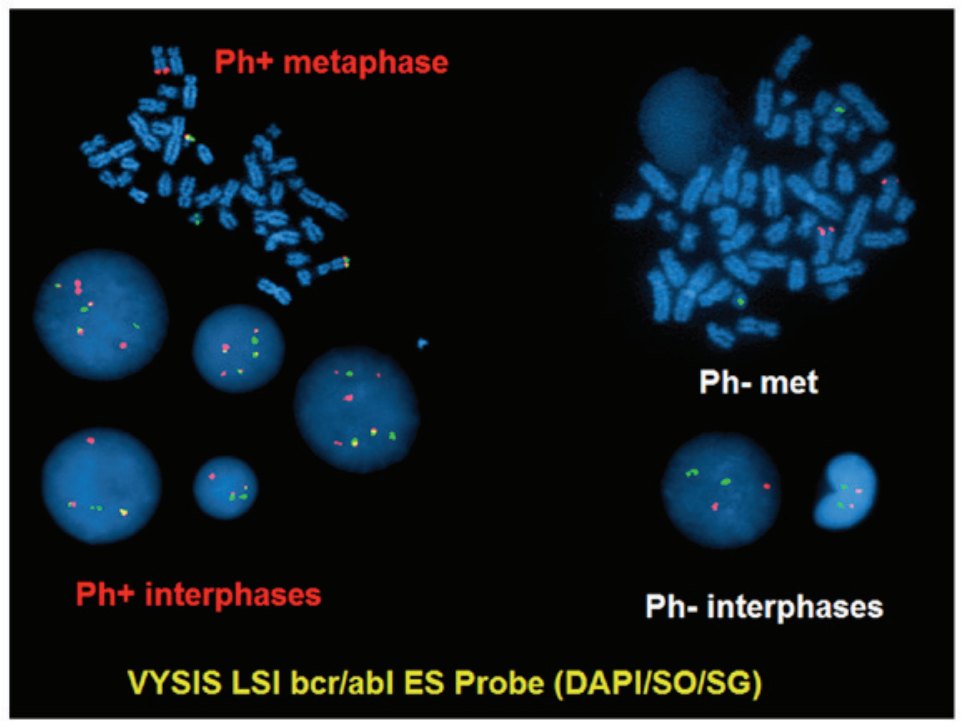

Figure 2. BCR-ABL1 fusion in bone marrow cells detected by fluorescence in situ hybridization. Ph, Philadelphia chromosome.

The physical examination identified a right inguinal node measuring $3 \times 4 \mathrm{~cm}$, whereas no cervical or axillary nodes were palpable. The patient was administered idarubicin, cytarabine, vincristine, dexamethasone and imatinib (600 mg/day) as initial treatment. After two courses of treatment, the patient achieved complete resolution of lymphadenopathy and complete cryptogenic response in the BM. The percentage of $B C R-A B L 1$ to $A B L 1$ transcripts decreased from 66.78 to 5.14 . However, after 3 more courses of treatment, the percentage of $B C R-A B L 1$ to $A B L 1$ transcripts exhibited no further decline. Therefore, chemotherapy was discontinued and the patient underwent related allogeneic hematopoietic stem cell transplant (Allo-HSCT) from her HLA-identical brother in September, 2006. Following engraftment (100\% donor), the patient achieved a major, but not complete, molecular remission (the percentage of $B C R-A B L 1$ to $A B L 1$ transcripts was 0.03-0.04). Four months after Allo-HSCT, the $B C R-A B L 1$ level increased to 0.7 . Therefore, imatinib $400 \mathrm{mg} / \mathrm{day}$ was administered. In June, 2007, the BM exhibited diploid female karyotypes and molecular studies revealed $90 \%$ of the cells to be donor. Blast cells (3\%) were found in the $\mathrm{BM}$, and FISH on BM examination detected no $B C R-A B L 1$ rearrangement. The patient underwent a series of donor lymphocyte infusions (DLIs) in August, 2007, February and June, 2008 and January, 2009. The imatinib dose was escalated to $800 \mathrm{mg} /$ day. However, the $B C R-A B L 1$ level 
remained between 0.28 and 0.65. In June, 2010, a fifth DLI was performed, following which the patient developed a mild continued skin graft-versus-host disease (GVHD). In September, 2010, imatinib was switched to dasatinib. From March, 2011 onwards, the patient switched to $100 \%$ donor cells and the BM showed no Philadelphia chromosome. The peripheral blood $B C R-A B L 1$ PCR was negative. Since then, the patient has remained in complete molecular remission and has been leukemia-free for $>48$ months.

\section{Discussion}

In $>95 \%$ chronic myeloid leukemia (CML) patients, the two major $B C R-A B L 1$ transcripts are $\mathrm{b} 2 \mathrm{a} 2$ and $\mathrm{b} 3 \mathrm{a} 2$, which encode the P210 oncoprotein. Very rarely, CML patients have the ela2 transcript, which encodes the $\mathrm{P} 190$ oncoprotein. In a large series study of 1,292 CML patients from the MD Anderson Cancer Center (1), only 14 patients expressed the ela2 transcript alone [9 in chronic phase (CP), 4 in accelerated phase (AP) and 1 in blast crisis (BC)]. Several studies from other countries (Korean and Mexican) also reported a similar incidence of the ela2 transcript, which was $\sim 1 \%$ in CML $(2,3)$. Thus, ela2 is a rare $B C R-A B L 1$ transcript in CML. Although an extramedullary BC may be observed in CML, mainly in CML-AP or BC, its presence in CML-CP is uncommon. Our CML patient with the ela2 $B C R-A B L 1$ transcript exhibited unusual characteristics at initial presentation of CML, namely manifesting an extramedullary BC. To the best of our knowledge, this is the first case report of CML with both the e1a2 $B C R-A B L 1$ transcript and extramedullary $\mathrm{BC}$ as initial presentation.

In addition to harboring the uncommon ela 2 transcript, this case had the notable characteristic of CML being atypical or occult. The peripheral blood count was within the normal range and the patient had no hepatosplenomegaly. In addition, the morphological examination of BM smear and biopsy revealed no abnormalities. However, the BM cytogenetics and FISH on BM confirmed the diagnosis of CML. It was reported that P190 BCR-ABL1 CML often exhibits monocytosis, with cytomorphological characteristics intermediate between CML and chronic myelomonocytic leukemia (4). However, our patient did not have monocytosis. The BM and blood tests indicated that CML was still in the early or occult stage, but the incidence of extramedullary $\mathrm{BC}$ demonstrated the aggressive characteristic of P190-positive CML. According to the World Health Organization recommendations, despite BM and blood status, extramedullary BC is a sign of CML BC (5).

This case also indicated that a complete and comprehensive BM examination is required in extramedullary MS cases. Indeed, MS is always accompanied by BM clonal disease. Even in certain 'isolated' MS cases, leukemic clones are already present in the BM. Although several groups have reported certain 'isolated' or 'aleukemic' MS cases without overt evidence of acute myeloid leukemia (AML), AML clones may be detected in the BM by cytogenetics and/or PCR-based methods $(6,7)$. Therefore, if extramedullary MS has been diagnosed, it is necessary to perform the abovementioned tests to definitively exclude leukemia associated with MS, even for those cases with 'normal' morphological characteristics.

The other characteristic of this case is the difficulty in management. Following treatment by a combination of traditional chemotherapy and imatinib, the extramedullary $\mathrm{BC}$ disappeared and patient achieved hematological and cytogenetic remission, along with minor molecular remission. Later on, after a series of treatments, including Allo-HSCT, DLI and second-generation tyrosine kinase inhibitor (TKI), a complete molecular remission was achieved after 7 years of continuous treatment. The optimal treatment for CML with the ela2 transcript has not been formulated, due to the rarity of this disease. TKIs have been proven to improve the outcome of CML patients, although the limited clinical data have shown that ela2 BCR-ABLCML exhibits a poorer response to first-line TKIs $(1,8)$. In a study by Verma et al (1), among 14 CML patients with only the ela2 transcript who received therapy containing imatinib, nilotinib or dasatinib, only 2 patients achieved a major molecular remission and only 6 patients ( 5 in $\mathrm{CP}$ and 1 in $\mathrm{AP}$ ) remained alive at a median of 39 months after diagnosis. That clinical study demonstrated that the efficacy of TKIs for P190 BCR-ABL1 CML is significantly lower compared with classic P210 BCR-ABL1 CML, whereas the clinical efficacy of new-generation TKIs, such as ponatinib, in ela2 $B C R-A B L 1 C M L$, is not yet certain. Jain et al reported two CML-CP patients with the ela2 transcript receiving ponatinib therapy (9). One patient achieved complete hematological remission, but never achieved a cytogenetic response, whereas the other patient achieved a minor cytogenetic response after 3 months of ponatinib therapy. Therefore, Allo-HSCT appears to be the best treatment option for CML at present. Our patient did not achieve a complete molecular remission following Allo-HSCT. However, after a series of DLIs, particularly after the last DLI, mild skin GVHD occurred, after which the patient switched to $100 \%$ donor cells and finally achieved complete molecular remission, which may be attributed, at least partially, to dasatinib.

According to our limited experience with this disease type, CML patients with the P190 oncoprotein always exhibit an aggressive disease course, poor response to TKI treatment and a high risk of transforming to AP and BC. The clinical characteristics and difficulties during the entire treatment course in this case are consistent with the abovementioned characteristics.

We consider this case to be very interesting and meaningful for hematologists and hematopathologists. First, to the best of our knowledge, this is the first report of an e1a2-P190 CML with extramedullary BC as initial presentation; and second, this case highlights the higher agressiveness of CML when $\mathrm{P} 190$ is present, and its refractoriness to treatment.

\section{References}

1. Verma D, Kantarjian HM, Jones D, Luthra R, Borthakur G, Verstovsek S, Rios MB and Cortes J: Chronic myeloid leukemia (CML) with P190 BCR-ABL: Analysis of characteristics, outcomes, and prognostic significance. Blood 114: 2232-2235, 2009.

2. Goh HG, Hwang JY, Kim SH, Lee YH, Kim YL and Kim DW: Comprehensive analysis of BCR-ABL transcript types in Korean CML patients using a newly developed multiplex RT-PCR. Transl Res 148: 249-256, 2006.

3. Arana-Trejo RM, Ruíz Sánchez E, Ignacio-Ibarra G, Báez de la Fuente E, Garces O, Gómez Morales E, Castro Granados M, Ovilla Martínez R, Rubio-Borja ME, Solís Anaya L, et al: BCR/ABL p210, p190 and p230 fusion genes in 250 Mexican patients with chronic myeloid leukaemia (CML). Clin Lab Haematol 24: 145-150, 2002. 
4. Melo JV, Myint H, Galton DA and Goldman JM: P190BCR-ABL chronic myeloid leukaemia: The missing link with chronic myelomonocytic leukaemia? Leukemia 8: 208-211, 1994.

5. Pileri SA, Orayi A and Falini B: Myeloid sarcoma. In: WHO Classification of Tumours of Haematopoietic Lymphoid Tissues Swerdlow S, Campo E, Harris NL, Jaffe ES, Pileri SA, Stein H, Thiele J and Vardiman JW (eds). Vol 2. 4th edition. IARC Press, Lyon, pp32-34, 2008.

6. Billio A, Pianezze G, Amato B and Fabris P: 'Isolated' peritoneal granulocytic sarcoma with molecular and chromosomal bone marrow involvement. Haematologica 87: EIM01, 2002.

7. Huang B, You P, Zhu P, DU Z, Wu B, Xu X and Chen Z: Isolated duodenal myeloid sarcoma associated with the $\mathrm{CBF} \beta / \mathrm{MYH} 11$ fusion gene followed by acute myeloid leukemia progression: A case report and literature review. Oncol Lett 8: 1261-1264, 2014
8. Pardanani A, Tefferi A, Litzow MR, Zent C, Hogan WJ, McClure RF and Viswanatha D: Chronic myeloid leukemia with p190BCR-ABL: Prevalence, morphology, tyrosine kinase inhibitor response, and kinase domain mutation analysis. Blood 114: 3502-3503, 2009.

9. Jain P, Romo CG, Khoury HJ, Kantarjian H and Cortes J: Clinical activity of ponatinib in patients with chronic myeloid leukemia in chronic phase with ela2 transcripts. Haematologica 98: e141-e142, 2013. 